\title{
Effects of spin on the dynamics of the 2D Dirac oscillator in the magnetic cosmic string background
}

\author{
Fabiano M. Andrade ${ }^{1, \mathrm{a}}$, Edilberto O. Silva ${ }^{2, \mathrm{~b}}$ \\ ${ }^{1}$ Departamento de Matemática e Estatística, Universidade Estadual de Ponta Grossa, Ponta Grossa, PR 84030-900, Brazil \\ 2 Departamento de Física, Universidade Federal do Maranhão, Campus Universitário do Bacanga, São Luís, MA 65085-580, Brazil
}

Received: 20 May 2014 / Accepted: 20 November 2014 / Published online: 10 December 2014

(C) The Author(s) 2014. This article is published with open access at Springerlink.com

\begin{abstract}
In this work the dynamics of a 2D Dirac oscillator in the spacetime of a magnetic cosmic string is considered. It is shown that earlier approaches to this problem have neglected a $\delta$ function contribution to the full Hamiltonian, which comes from the Zeeman interaction. The inclusion of spin effects leads to results which confirm a modified dynamics. Based on the self-adjoint extension method, we determined the most relevant physical quantities, such as energy spectrum, wave functions and the self-adjoint extension parameter by applying boundary conditions allowed by the system.
\end{abstract}

\section{Introduction}

The Dirac oscillator is a natural model for studying properties of physical systems. This model is based on the dynamics of a harmonic oscillator for spin-1/2 particles by introducing a nonminimal prescription into free Dirac equation [1]. Because it is an exactly solvable model, several investigations have been developed in the context of this theoretical framework in the last years. The interest in this issue appears in different contexts, such as quantum optics [2-4], supersymmetry [5-7], nuclear reactions [8], Clifford algebra $[9,10]$, and noncommutative space [11,12]. Recently, the one-dimensional Dirac oscillator has been verified experimentally by J. A. Franco-Villafañe et al., based on a tightbinding system [13]. A detailed description for the Dirac oscillator is given in Ref. [14] and for other contributions see Refs. [15-22].

Among the various contexts in which the Dirac oscillator can be addressed, we refer to the cosmic string, a linear defect that change the topology of the medium when viewed globally. This framework has inspired a great deal

\footnotetext{
a e-mail: fmandrade@uepg.br

b e-mail: edilbertoo@gmail.com
}

of investigation in recent years. Such works encompass several distinct aspects to investigate the effects produced by topological defects of this nature [23-29].

In this work, we generalize the results in [17] for a 2D Dirac oscillator in the magnetic cosmic string background showing rigorously how the dynamics of this system is affected when the effects of spin are taken into account. Our approach is based on the self-adjoint extension method which is appropriate to address any system endowed with a singular Hamiltonian (due to localized field sources or quantum confinement). We determine the most relevant physical quantities from the present model, such as energy spectrum, wave functions, and self-adjoint extension parameter by applying boundary conditions allowed by the system.

\section{The 2D Dirac oscillator in the magnetic cosmic string background}

In this section, we study the motion of the particle in the magnetic cosmic string background. The cosmic string spacetime with an internal magnetic field is an object described by the following line element in cylindrical coordinates $(t, r, \varphi, z)$ :

$d s^{2}=-d t^{2}+d r^{2}+\alpha^{2} r^{2} d \varphi^{2}+d z^{2}$,

with $-\infty<(t, z)<\infty, r \geq 0$, and $0 \leq \varphi \leq 2 \pi$. The parameter $\alpha$ is related to the linear mass density $\tilde{m}$ of the string by $\alpha=1-4 \tilde{m}$ runs in the interval $(0,1]$ and corresponds to a deficit angle $\gamma=2 \pi(1-\alpha)$. Geometrically, the metric (1) corresponds to a Minkowiski spacetime with a conical singularity [30].

We begin with the Dirac equation in the curved spacetime (with $\hbar=c=1$ ):

$\left[i \gamma^{\mu}\left(\partial_{\mu}+\Gamma_{\mu}\right)-e \gamma^{\mu} A_{\mu}-M\right] \Psi=0$,

where $e$ is the charge, $M$ is mass of the particle, $\Psi$ is a four-component spinorial wave function, and $\Gamma_{\mu}$ is the spinor 
affine connection, which is given by [31,32]

$\Gamma_{\mu}=\frac{1}{4} i \omega_{\mu \bar{a} \bar{b}} \sigma_{\bar{a} \bar{b}}=-\frac{1}{8} \omega_{\mu \bar{a} \bar{b}}\left[\gamma^{\bar{a}}, \gamma^{\bar{b}}\right]$,

where $\gamma^{\bar{a}}$ are the standard Dirac matrices in Minkowski spacetime and $\omega_{\mu \bar{a} \bar{b}}$ is the spin connection, given by

$\omega_{\mu \bar{a} \bar{b}}=\eta_{\bar{a} c} e_{\nu}^{\bar{c}} e_{\bar{b}}^{\sigma} \Gamma_{\sigma \mu}^{\nu}-\eta_{\bar{a} \bar{c}} e_{\nu}^{\bar{c}} \partial_{\mu} e_{\bar{b}}^{v}$,

with $(\mu, v)=(0,1,2,3)$ and $(\bar{a}, \bar{b})=(0,1,2,3) ; \Gamma_{\sigma \mu}^{\nu}$ is the Christoffel symbol, $\eta^{\bar{a}} \bar{b}$ is the metric tensor, $e_{\bar{a}}^{\nu}$ is the basis tetrad which will be defined below. The spin connection (3) allows us to construct a local frame through the basis tetrad which gives the spinors in the curved spacetime. Also, the $\gamma^{\mu}$ matrices are the generalized Dirac matrices defining the covariant Clifford algebra,

$\left\{\gamma^{\mu}, \gamma^{\nu}\right\}=2 g^{\mu \nu}$

and are written in terms of the standard Dirac matrices $\gamma^{\bar{a}}$ in Minkowski spacetime as

$\gamma^{\mu}=e_{\bar{a}}^{\mu} \gamma^{\bar{a}}$

with $\gamma^{\bar{a}}=\left(\gamma^{\overline{0}}, \gamma^{\bar{\imath}}\right)$, and

$\gamma^{\overline{0}}=\left(\begin{array}{ll}\mathbb{1} & 0 \\ 0 & -\mathbb{1}\end{array}\right), \quad \gamma^{\bar{\imath}}=\left(\begin{array}{ll}0 & \sigma^{i} \\ -\sigma^{i} & 0\end{array}\right) \quad(i=1,2,3)$,

where $\sigma^{i}$ are the standard Pauli matrices and $\mathbb{1}$ is the $2 \times 2$ identity matrix. The basis tetrad $e_{\bar{a}}^{\mu}$ in Eq. (6) is chosen to be [17]

$e_{\bar{a}}^{\mu}=\left(\begin{array}{llll}1 & 0 & 0 & 0 \\ 0 & \cos \varphi & \sin \varphi & 0 \\ 0 & -\sin \varphi / \alpha r & \cos \varphi / \alpha r & 0 \\ 0 & 0 & 0 & 1\end{array}\right)$,

satisfying the condition

$e_{\bar{a}}^{\mu} e_{\frac{v}{b}} \eta^{\bar{a} \bar{b}}=g^{\mu \nu}$

The matrices $\gamma^{\mu}$ in Eq. (6) are given more explicitly as

$\gamma^{0}=e_{0}^{t} \gamma^{\overline{0}} \equiv \gamma^{t}$

$\gamma^{3}=e_{\overline{0}}^{z} \gamma^{\overline{0}} \equiv \gamma^{z}$

$\gamma^{1}=e_{\bar{a}}^{1} \gamma^{\bar{a}} \equiv \gamma^{r}$,

$\gamma^{r}=e_{\overline{0}}^{r} \gamma^{\overline{0}}+e_{\overline{1}}^{r} \gamma^{\overline{1}}+e_{\overline{2}}^{r} \gamma^{\overline{2}}=\gamma^{\overline{1}} \cos \varphi+\gamma^{\overline{2}} \sin \varphi$,

$\gamma^{2}=e_{\bar{a}}^{2} \gamma^{\bar{a}} \equiv \frac{\gamma^{\varphi}}{\alpha r}$

$\gamma^{\varphi}=e_{\overline{0}}^{\varphi} \gamma^{\overline{0}}+e_{\overline{1}}^{\varphi} \gamma^{\overline{1}}+e_{\overline{2}}^{\varphi} \gamma^{\overline{2}}=-\gamma^{\overline{1}} \sin \varphi+\gamma^{\overline{2}} \cos \varphi$.

The starting point for the derivation of Eq. (3) is that the curved-space gamma matrices are covariantly constant [3133], i.e., $\nabla_{\mu} \gamma^{\lambda}=0$ (see Appendix A). For the specific basis tetrad (8), the connection is found to be

$\boldsymbol{\Gamma}=\left(0,0, \Gamma_{\varphi}, 0\right)$,

with the non-vanishing element given as

$\Gamma_{\varphi}=-\frac{1}{2}(1-\alpha) \gamma_{\overline{1}} \gamma_{\overline{2}}$.

Details for the calculation of the connection are given in Appendix B.

According to the tetrad postulated [31-34], the matrices $\gamma^{\bar{a}}$ could be any set of constant Dirac matrices. Thus, we are free to choose a representation for the matrices $\gamma^{\bar{a}}$. Making use of the symmetry under $\mathrm{z}$ translations of the system, we can reduce the four-component Dirac equation (2) to two two-component spinor equations. To do this, we consider the vector potential A as being intrinsically two-dimensional, i.e., it has only two components and depends on only two spatial coordinates, and we take $p_{z}=z=0$. In this manner, the relevant equation is

$[\tilde{\beta} \tilde{\gamma} \cdot \pi+\tilde{\beta} M] \psi=E \psi$,

where $\psi$ is a two-component spinor and

$\pi=-i\left(\nabla_{\alpha}+\Gamma\right)-e \mathbf{A}$

is the generalized momentum,

$\nabla_{\alpha}=\frac{\partial}{\partial r} \hat{\mathbf{r}}+\frac{1}{\alpha r} \frac{\partial}{\partial \varphi} \hat{\boldsymbol{\varphi}}$

is the gradient operator in polar coordinates, and the $\tilde{\gamma}^{\bar{a}}$ matrices are given in terms of the Pauli matrices as

$\tilde{\beta}=\tilde{\gamma}^{\overline{0}}=\sigma^{z}, \quad \tilde{\beta} \tilde{\gamma}^{\overline{1}}=\sigma^{1}, \quad \tilde{\beta} \tilde{\gamma}^{\overline{2}}=s \sigma^{2}$,

where the parameter $s$, which has a value of twice the spin value, can be introduced to characterizing the two spin states $[35,36]$, with $s=+1$ for spin "up" and $s=-1$ for spin "down" [37].

In the representation (16), Eq. (12) yields

$\Gamma_{\varphi}=-\frac{1}{2}(1-\alpha) i \sigma_{2}\left(-i s \sigma_{1}\right)=i \frac{(1-\alpha)}{2} s \sigma^{z}$.

The magnetic vector potential in polar coordinates in the Coulomb gauge is chosen to be

$e \mathbf{A}=-\frac{\phi}{\alpha r} \hat{\boldsymbol{\varphi}}$

where $\phi=\Phi / \Phi_{0}$ is the flux parameter with $\Phi_{0}=2 \pi / e$ $((\hat{\mathbf{r}}, \hat{\boldsymbol{\varphi}})$ denote the unit vectors in polar coordinates.) This choice for the vector potential gives a magnetic flux tube, in the background space described by the metric (1), coinciding with the cosmic string and with the magnetic field strength given by

$e B=-\frac{\phi}{\alpha} \frac{\delta(r)}{r}$. 
Note that, in the limit as $\alpha \rightarrow 1$, we obtain the magnetic field in Euclidean space.

The 2D Dirac oscillator is introduced by the non-minimal substitution [1]

$\frac{1}{i} \nabla_{\alpha} \rightarrow \frac{1}{i} \nabla_{\alpha}-i M \omega \beta \mathbf{r}$,

where $\mathbf{r}$ is the position vector and $\omega$ the frequency of the oscillator (for a comprehensive discussion of the Dirac oscillator see Ref. [14]). In this case, Eq. (13) reads

$[\boldsymbol{\alpha} \cdot(\boldsymbol{\pi}-i M \omega \beta \mathbf{r})+\beta M] \psi=E \psi$.

The second order equation implied by Eq. (21) is obtained by applying the matrix operator

$[\beta M+E+\boldsymbol{\alpha} \cdot(\boldsymbol{\pi}-i M \omega \beta \mathbf{r})]$.

So, one finds

$\left(E^{2}-M^{2}\right) \psi=[\boldsymbol{\alpha} \cdot(\boldsymbol{\pi}-i M \omega \beta \mathbf{r})][\boldsymbol{\alpha} \cdot(\boldsymbol{\pi}-i M \omega \beta \mathbf{r})] \psi$.

Inserting Eqs. (19), (18), and the expression for $\Gamma_{\varphi}$ in (17) into Eq. (23), one obtains

$\left(E^{2}-M^{2}\right) \psi=H \psi$,

where

$$
\begin{aligned}
H= & {\left[-i \nabla_{\alpha}+\left(\frac{\phi}{\alpha}+\frac{1-\alpha}{2 \alpha} s \sigma^{z}\right) \frac{1}{r} \hat{\varphi}\right]^{2} } \\
& -2 M \omega\left[\sigma^{z}+s\left(\frac{1}{i \alpha} \frac{\partial}{\partial \varphi}+\frac{\phi}{\alpha}+\frac{1-\alpha}{2 \alpha} s \sigma^{z}\right)\right] \\
& +M^{2} \omega^{2} r^{2}+\frac{\phi s}{\alpha} \frac{\delta(r)}{r} \sigma^{z} .
\end{aligned}
$$

In Eq. (25), the quantity

$\frac{\phi}{\alpha}+\frac{1-\alpha}{2 \alpha} s \sigma^{z}$

contributes to the term which depends explicitly on the spin of the particle. The first term is the contribution due to the magnetic flux while the second is due to the spin connection. Note that, by making $\alpha=1$ (flat spacetime) and $\phi=0$ (absence of a magnetic field) in Eq. (24), we obtain, for the planar case, the 2D Dirac oscillator as proposed by Moshinsky and Szczepaniak [1] and discussed in Appendix C.

Making use of the underlying rotational symmetry we can express the two-component spinor as

$\psi(r, \varphi)=\left(\begin{array}{l}\psi_{1} \\ \psi_{2}\end{array}\right)=\left(\begin{array}{l}f_{m}(r) e^{i m \varphi} \\ g_{m}(r) e^{i(m+s) \varphi}\end{array}\right)$,

with $m \in \mathbb{Z}$. By replacing Eq. (27) into Eq. (24), we obtain the radial equation for $f_{m}(r)$

$H f_{m}(r)=k^{2} f_{m}(r)$, where

$k^{2}=E^{2}-M^{2}+2 M \omega(s j+1)$,

$j=\frac{1}{\alpha}\left(m+\phi+\frac{1-\alpha}{2} s\right)$,

$H=H_{0}+\frac{\phi s}{\alpha} \frac{\delta(r)}{r}$,

and

$H_{0}=-\frac{d^{2}}{d r^{2}}-\frac{1}{r} \frac{d}{d r}+\frac{j^{2}}{r^{2}}+M^{2} \omega^{2} r^{2}$.

The Hamiltonian in Eq. (31) governs the dynamics of a Dirac oscillator in a magnetic cosmic string background, i.e., a Dirac oscillator problem in the presence of the AharonovBohm effect in a conical spacetime. The presence of a twodimension $\delta$ interaction in the radial Hamiltonian $H$, which is singular at the origin, makes the problem more complicated to solve. The most adequate manner to address this kind of point interaction potential is by making use of the self-adjoint extension approach $[38,39]$. This is the method adopted in this work and discussed in the next section.

\section{Self-adjoint extension analysis}

In this section, we review some concepts on the self-adjoint extension approach. An operator $\mathscr{O}$, with domain $\mathscr{D}(\mathscr{O})$, is said to be self-adjoint if and only if $\mathscr{O}=\mathscr{O}^{\dagger}$ and $\mathscr{D}(\mathscr{O})=\mathscr{D}\left(\mathscr{O}^{\dagger}\right), \mathscr{O}^{\dagger}$ being the adjoint of operator $\mathscr{O}$. For smooth functions, $\xi \in C_{0}^{\infty}\left(\mathbb{R}^{2}\right)$ with $\xi(0)=0$, we should have $H \xi=H_{0} \xi$, and it is possible to interpret the Hamiltonian (31) as a self-adjoint extension of $\left.H_{0}\right|_{C_{0}^{\infty}\left(\mathbb{R}^{2} /\{0\}\right)}[40$ 42]. The self-adjoint extension approach consists, essentially, in extending the domain of $\mathscr{D}(\mathscr{O})$ in order to match $\mathscr{D}\left(\mathscr{O}^{\dagger}\right)$. From the theory of symmetric operators, it is a wellknown fact that the symmetric radial operator $H_{0}$ is essentially self-adjoint for $|j| \geq 1$, while for $|j|<1$ it admits an one-parameter family of self-adjoint extensions [43], $H_{0, \lambda_{m}}$, where $\lambda_{m}$ is the self-adjoint extension parameter. To characterize this family, we will use the approach in $[38,39]$, which is based on the boundary conditions at the origin. All the self-adjoint extensions $H_{0, \lambda_{m}}$ of $H_{0}$ are parametrized by the boundary condition at the origin

$\Omega_{0}=\lambda_{m} \Omega_{1}$,

with

$\Omega_{0}=\lim _{r \rightarrow 0^{+}} r^{|j|} f_{m}(r)$,

$\Omega_{1}=\lim _{r \rightarrow 0^{+}} \frac{1}{r^{|j|}}\left[f_{m}(r)-\Omega_{0} \frac{1}{r^{|j|}}\right]$,

where $\lambda_{m} \in \mathbb{R}$ is the self-adjoint extension parameter. For $\lambda_{m}=0$, we have the free Hamiltonian (without the $\delta$ 
function) with regular wave functions at the origin, and for $\lambda_{m} \neq 0$ the boundary condition in Eq. (33) permit an $r^{-|j|}$ singularity in the wave functions at the origin.

\section{The bound state energy and wave function}

In this section, we determine the energy spectrum for the Dirac oscillator in the cosmic string background by solving Eq. (28). For $r \neq 0$, the equation for the component $f_{m}(r)$ can be transformed by the variable change $\rho=M \omega r^{2}$ resulting in

$\rho f_{m}^{\prime \prime}(\rho)+f_{m}^{\prime}(\rho)-\left(\frac{j^{2}}{4 \rho}+\frac{\rho}{4}-\frac{k^{2}}{4 \gamma}\right) f_{m}(\rho)=0$,

with $\gamma=M \omega$. Due to the boundary condition in Eq. (33), we seek regular and irregular solutions for Eq. (36). Studying the asymptotic limits of Eq. (36) leads us to the following regular $(+)$ (irregular $(-)$ ) solution:

$f_{m}(\rho)=\rho^{ \pm|j| / 2} e^{-\rho / 2} F(\rho)$.

With this, Eq. (36) is rewritten as

$$
\begin{aligned}
& \rho F^{\prime \prime}(\rho)+(1 \pm|j|-\rho) F^{\prime}(\rho) \\
& \quad-\left(\frac{1 \pm|j|}{2}-\frac{k^{2}}{4 \gamma}\right) F(\rho)=0 .
\end{aligned}
$$

Equation (36) is of the confluent hypergeometric equation type

$z F^{\prime \prime}(z)+(b-z) F^{\prime}(z)-a F(z)=0$.

In this manner, the general solution for Eq. (36) is

$$
\begin{aligned}
f_{m}(r)= & a_{m} \rho^{|j| / 2} e^{-\rho / 2} F\left(d_{+}, 1+|j|, \rho\right) \\
& +b_{m} \rho^{-|j| / 2} e^{-\rho / 2} F\left(d_{-}, 1-|j|, \rho\right),
\end{aligned}
$$

with

$d_{ \pm}=\frac{1 \pm|j|}{2}-\frac{k^{2}}{4 \gamma}$.

In Eq. (40), $F(a, b, z)$ is the confluent hypergeometric function of the first kind [44] and $a_{m}$ and $b_{m}$ are, respectively, the coefficients of the regular and irregular solutions.

In this point, we apply the boundary condition in Eq. (33). Doing this, one finds the following relation between the coefficients $a_{m}$ and $b_{m}$ :

$\lambda_{m} \gamma^{|j|}=\frac{b_{m}}{a_{m}}\left[1+\frac{\lambda_{m} k^{2}}{4(1-|j|)} \lim _{r \rightarrow 0^{+}} r^{2-2|j|}\right]$.

We note that $\lim _{r \rightarrow 0^{+}} r^{2-2|j|}$ diverges if $|j| \geq 1$. This condition implies that $b_{m}$ must be zero if $|j| \geq 1$ and only the regular solution contributes to $f_{m}(r)$. For $|j|<1$, when the operator $H_{0}$ is not self-adjoint, there arises a contribution of the irregular solution to $f_{m}(r)[29,37,45-49]$. In this manner, the contribution of the irregular solution for the system wave function stems from the fact that the operator $H_{0}$ is not self-adjoint.

For $f_{m}(r)$ be a bound state wave function, it must vanish at large values of $r$, i.e., it must be normalizable. So, from the asymptotic representation of the confluent hypergeometric function, the normalizability condition is translated in

$\frac{b_{m}}{a_{m}}=-\frac{\Gamma(1+|j|)}{\Gamma(1-|j|)} \frac{\Gamma\left(d_{-}\right)}{\Gamma\left(d_{+}\right)}$.

From Eq. (42), for $|j|<1$ we have $b_{m} / a_{m}=\lambda_{m} \gamma^{|j|}$. Using this result in Eq. (43), one finds

$\frac{\Gamma\left(d_{+}\right)}{\Gamma\left(d_{-}\right)}=-\frac{1}{\lambda_{m} \gamma^{|j|}} \frac{\Gamma(1+|j|)}{\Gamma(1-|j|)}$.

Equation (44) implicitly determines the bound state energy for the Dirac oscillator in the cosmic string background for different values of the self-adjoint extension parameter. Two limiting values for the self-adjoint extension parameter deserve some attention. For $\lambda_{m}=0$, when the $\delta$ interaction is absent, only the regular solution contributes for the bound state wave function. On the other side, for $\lambda_{m}=\infty$ only the irregular solution contributes for the bound state wave function. For all other values of the self-adjoint extension parameter, both regular and irregular solutions contribute for the bound state wave function. The energies for the limiting values are obtained from the poles of the gamma function, namely,

$\begin{cases}d_{+}=-n & \text { for } \lambda_{m}=0 \quad \text { (regular solution) } \\ d_{-}=-n & \text { for } \lambda_{m}=\infty \text { (irregular solution) }\end{cases}$

with $n$ a nonnegative integer, $n=0,1,2, \ldots$ By manipulation of Eq. (45), we obtain

$$
\begin{aligned}
E= & \pm\left\{M^{2}+2 M \omega\left[2 n \pm \frac{1}{\alpha}\left|m+\phi+\frac{1-\alpha}{2} s\right|\right.\right. \\
& \left.\left.-\frac{s}{\alpha}\left(m+\phi+\frac{1-\alpha}{2} s\right)\right]\right\}^{1 / 2} .
\end{aligned}
$$

In particular, it should be noted that for the case when $|j| \geq 1$ or when the $\delta$ interaction is absent, only the regular solution contributes for the bound state wave function $\left(b_{m}=0\right)$, and the energy is given by Eq. (46) using the plus sign. Note that, for $\alpha=1$ (flat space) and $\phi=0$ (no magnetic flux), Eq. (46) coincides with the energy found for the usual 2D Dirac oscillator (cf. Eq. (C.35) in C). Without loss of generality, let us suppose $0<\phi<1[42,50,51]$. In this interval, and recalling that we are interested in the case where $0<\alpha \leq 1$, another interesting feature is present in the energy eigenvalues. For the regular solution, the eigenvalues are independent of $m, \phi$, and $\alpha$ for $s=1$. This situation is shown in Fig. 1a for $n=1$ and $m=1$. However, this independence is absent for $s=-1$, as shown in Fig. $1 \mathrm{~b}$ for $n=1$ and $m=1$. In the other hand, for the irregular solution, the eigenvalues are independent of $m, \phi$ and $\alpha$ for $s=-1$ and dependent 

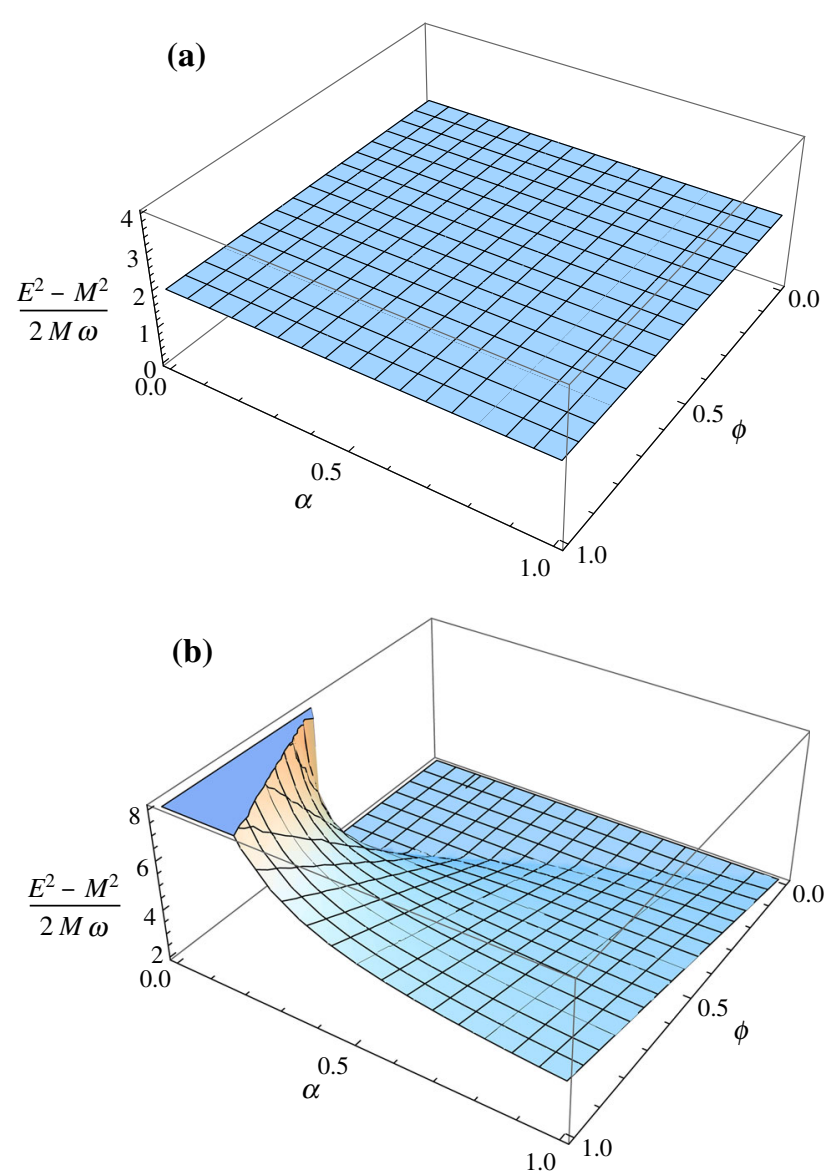

Fig. 1 The energy eigenvalues for the regular solution for a 2D Dirac oscillator in the cosmic string background as a function of the declination $\alpha$ and the magnetic flux $\phi$ for $n=1, m=1$, and for $\mathbf{a} s=1$ and b $s=-1$. For convenience, we plot $\left(E^{2}-M^{2}\right) / 2 M \omega$ instead $E$

for $s=1$. Also, for $s=-1$, decreasing the value of $\alpha$, the energy increase as an effect of the quantum localization.

The unnormalized bound state wave functions for our problem are

$f_{m}(r)=\rho^{ \pm|j| / 2} e^{-\rho / 2} F(-n, 1 \pm|j|, \rho)$.

The self-adjoint extension is related with the presence of the $\delta$ interaction. In this manner, the self-adjoint extension parameter must be related with the $\delta$ interaction coupling constant $\phi s / \alpha$. In fact, as shown in Refs. [28,29] (see also Refs. [45,52]), from the regularization of the $\delta$ interaction, it is possible to find such a relationship. Using the regularization method, one obtains the following equation for the bound state energy:

$\frac{\Gamma\left(d_{+}\right)}{\Gamma\left(d_{-}\right)}=-\frac{1}{r_{0}^{2|j|}}\left(\frac{\phi s+\alpha|j|}{\phi s-\alpha|j|}\right) \frac{1}{\gamma^{|j|}} \frac{\Gamma(1+|j|)}{\Gamma(1-|j|)}$.

By comparing Eqs. (44) and (48), this relation is found to be

$\frac{1}{\lambda_{m}}=\frac{1}{r_{0}^{2|j|}}\left(\frac{\phi s+\alpha|j|}{\phi s-\alpha|j|}\right)$ where $r_{0}$ is a very small radius which comes from the $\delta$ regularization $[28,29]$.

\section{Nonrelativisitic limit}

We shall now take the nonrelativistic limit of Eq. (24). Using $E=M+\mathscr{E}$ with $M \gg \mathscr{E}$, we obtain

$2 M \mathscr{E} \psi=H \psi$

Performing the same steps as for the relativistic case, one obtains the shifted energy levels (cf. Appendix C)

$$
\begin{aligned}
\mathscr{E}+\omega= & {\left[1+2 n \pm \frac{1}{\alpha}\left|m+\phi+\frac{1-\alpha}{2} s\right|\right.} \\
& \left.-\frac{s}{\alpha}\left(m+\phi+\frac{1-\alpha}{2} s\right)\right] \omega .
\end{aligned}
$$

In this equation, the $+(-)$ sign is for $\lambda_{m}=0\left(\lambda_{m}=\infty\right)$ when one has regular (irregular) solution. We note that the energy in Eq. (51) corresponds to equation (54) of Ref. [53] (cf. also Eq. (C.37) in Appendix C) with two additional contributions, the spin-orbit coupling and the spin connection.

\section{Conclusions}

In this contribution, we have addressed the Dirac oscillator interacting with a topological defect and in the presence of the Aharonov-Bohm potential. This system has been studied in Ref. [17]. However, the authors do not take into account the effects of spin. In other words, the term proportional to the $\delta$ interaction was discarded, by considering only the regular solution of the problem. The presence of this term has direct implications in the energy spectrum and wave functions of the oscillator. The correct approach to this problem must include spin effects, which are explicitly manifested by the spinorbit coupling term, and so we have a complete description for the dynamics of the 2D Dirac oscillator. We consider the self-adjoint extension method and show that the spin-orbit coupling term, which results in a $\delta$ interaction, cannot be dropped from the Hamiltonian. Although being singular at the origin, this term reveals that both regular and irregular solutions contribute for the bound state wave function and, consequently, for the energy spectrum. Expressions for the bound states energy for different values of the self-adjoint extension parameter were obtained. For two specific values for the self-adjoint extension parameter, i.e., $\lambda_{m}=0$ and $\lambda_{m}=\infty$, the bound state energies are given explicitly in Eq. (46). We also verified that, for the flat space $(\alpha=1)$ and no magnetic flux $(\phi=0)$, the results of the usual 2D Dirac oscillator are recovered. 
Acknowledgments We would like to thank R. Casana and L. R. B. Castro for fruitful discussions. This work was supported by the Fundação Araucária (Grant No. 205/2013 (PPP) and No. 484/2014 (PQ)), and the Conselho Nacional de Desenvolvimento Científico e Tecnológico (Grants No. 482015/2013-6 (Universal), No. 306068/2013-3 (PQ)) and FAPEMA (Grant No. 00845/13). Finally, we acknowledge some suggestions made by the anonymous referees in order to improve the present work.

Open Access This article is distributed under the terms of the Creative Commons Attribution License which permits any use, distribution, and reproduction in any medium, provided the original author(s) and the source are credited.

Funded by $\mathrm{SCOAP}^{3}$ / License Version CC BY 4.0.

\section{Appendix A: Covariancy of $\gamma$ matrices in the curved spacetime}

In this appendix, we give the details of the calculation of the covariant derivative of $\gamma^{\mu}$ in Eq. (6). As a consequence of the tetrad we postulate

$\nabla_{\mu} e_{\nu}^{\bar{a}} \equiv \partial_{\mu} e_{\nu}^{\bar{a}}-e_{\sigma}^{\bar{a}} \Gamma_{\mu \nu}^{\sigma}+\omega_{\mu \bar{b}}^{\bar{a}} e_{\nu}^{\bar{b}}=0$,

together with the condition

$\left[\gamma^{\bar{a}}, \Gamma_{\mu}\right]=\omega_{\mu \bar{b}}^{\bar{a}} \gamma^{\bar{b}}$

we have

$\nabla_{\mu} \gamma^{\lambda}=\partial_{\mu} \gamma^{\lambda}+\Gamma_{\mu \nu}^{\lambda} \gamma^{\nu}+\left[\gamma^{\lambda}, \Gamma_{\mu}\right]=0$.

To check this, first we need to find the relevant Christoffel symbols. They are found to be

$\Gamma_{\varphi \varphi}^{r}=-\alpha^{2} r$

$\Gamma_{r \varphi}^{\varphi}=\Gamma_{\varphi r}^{\varphi}=\frac{1}{r}$

Moreover, we also make use of Eqs. (10a)-(10e) and Eq. (A.3) and then calculate for each $(\mu, \lambda=t, r, \varphi)$. Then, for $\mu=t$, we write

$\nabla_{t} \gamma^{\lambda}=\partial_{t} \gamma^{\lambda}+\Gamma_{t v}^{\lambda} \gamma^{v}+\left[\gamma^{\lambda}, \Gamma_{t}\right] \quad(\mu=t)$.

Since $\Gamma_{t v}^{t}=\Gamma_{t}=\partial_{t} \gamma^{t}=0, \Gamma_{t v}^{r}=\partial_{t} \gamma^{r}=0$, and $\Gamma_{t v}^{\varphi}=$ $\partial_{t} \gamma^{\varphi}=0$, it follows that

$\nabla_{t} \gamma^{t}=\partial_{t} \gamma^{t}+\Gamma_{t v}^{t} \gamma^{v}+\left[\gamma^{t}, \Gamma_{t}\right]=0 \quad(\lambda=t)$,

$\nabla_{t} \gamma^{r}=\partial_{t} \gamma^{r}+\Gamma_{t \nu}^{r} \gamma^{\nu}+\left[\gamma^{r}, \Gamma_{t}\right]=0 \quad(\lambda=r)$,

$\nabla_{t} \gamma^{\varphi}=\partial_{t} \gamma^{\varphi}+\Gamma_{t \nu}^{\varphi} \gamma^{\nu}+\left[\gamma^{\varphi}, \Gamma_{t}\right]=0 \quad(\lambda=\varphi)$,

so that

$\nabla_{t} \gamma^{\lambda}=\nabla_{t} \gamma^{t}+\nabla_{t} \gamma^{r}+\nabla_{t} \gamma^{\varphi}=0 \quad(\lambda=t, r, \varphi)$.

Next, we have

$\nabla_{r} \gamma^{\lambda}=\partial_{r} \gamma^{\lambda}+\Gamma_{r \nu}^{\lambda} \gamma^{\nu}+\left[\gamma^{\lambda}, \Gamma_{r}\right] \quad(\mu=r)$, and by using $\Gamma_{r \varphi}^{\varphi}=1 / r($ with $v=\varphi), \Gamma_{r v}^{t}=\Gamma_{r v}^{r}=0$, $\partial_{t} \gamma^{t}=\partial_{r} \gamma^{r}=0$, we get

$$
\begin{aligned}
\nabla_{r} \gamma^{t}= & \partial_{r} \gamma^{t}+\Gamma_{r \nu}^{t} \gamma^{\nu}+\left[\gamma^{t}, \Gamma_{r}\right]=0 \quad(\lambda=t), \\
\nabla_{r} \gamma^{r}= & \partial_{r} \gamma^{r}+\Gamma_{r \nu}^{r} \gamma^{\nu}+\left[\gamma^{r}, \Gamma_{r}\right]=0 \quad(\lambda=r), \\
\nabla_{r} \gamma^{\varphi}= & \partial_{r} \gamma^{\varphi}+\Gamma_{r \nu}^{\varphi} \gamma^{\nu}+\left[\gamma^{\varphi}, \Gamma_{r}\right] \\
= & \frac{1}{\alpha r^{2}}\left(\gamma^{\overline{1}} \sin \varphi-\gamma^{2} \cos \varphi\right) \\
& +\frac{1}{\alpha r^{2}}\left(-\gamma^{\overline{1}} \sin \varphi+\gamma^{\overline{2}} \cos \varphi\right) \\
= & 0 \quad(\lambda=\varphi),
\end{aligned}
$$

and consequently

$\nabla_{r} \gamma^{\lambda}=\nabla_{r} \gamma^{t}+\nabla_{r} \gamma^{r}+\nabla_{r} \gamma^{\varphi}=0 \quad(\lambda=t, r, \varphi)$.

Now, for $\mu=\varphi$, we write

$\nabla_{\varphi} \gamma^{\lambda}=\partial_{\varphi} \gamma^{\lambda}+\Gamma_{\varphi \nu}^{\lambda} \gamma^{\nu}+\left[\gamma^{\lambda}, \Gamma_{\varphi}\right] \quad(\mu=\varphi)$,

and again, since $\partial_{\varphi} \gamma^{t}=\Gamma_{\varphi v}^{t}=0$, and using Eqs. (A.4) and (A.5), we have

$$
\begin{aligned}
\nabla_{\varphi} \gamma^{t}= & \partial_{\varphi} \gamma^{t}+\Gamma_{\varphi \nu}^{t} \gamma^{\nu}-\left[\Gamma_{\varphi}, \gamma^{t}\right] \\
= & \frac{1}{2}(1-\alpha) \gamma_{\overline{1}} \gamma_{\overline{2}} \gamma^{t}-\frac{1}{2}(1-\alpha) \gamma^{t} \gamma_{\overline{1}} \gamma_{\overline{2}} \\
= & 0 \quad(\lambda=t), \\
\nabla_{\varphi} \gamma^{r}= & \partial_{\varphi} \gamma^{r}+\Gamma_{\varphi \nu}^{r} \gamma^{\nu}-\left[\Gamma_{\varphi}, \gamma^{r}\right] \\
= & -(1-\alpha) \gamma^{\overline{2}} \cos \varphi-(1-\alpha) \gamma^{\overline{1}} \sin \varphi \\
& +(1-\alpha) \gamma^{\overline{2}} \cos \varphi-(1-\alpha) \gamma^{\overline{1}} \sin \varphi \\
= & 0 \quad(\lambda=r),
\end{aligned}
$$

$$
\begin{aligned}
\nabla_{\varphi} \gamma^{\varphi}= & \partial_{\varphi} \gamma^{\varphi}+\Gamma_{\varphi \nu}^{\varphi} \gamma^{\nu}-\left[\Gamma_{\varphi}, \gamma^{\varphi}\right] \\
= & -\frac{1}{\alpha r}(1-\alpha)\left(\gamma^{\overline{1}} \cos \varphi+\gamma^{\overline{2}} \sin \varphi\right) \\
& +\frac{1}{\alpha r}(1-\alpha)\left(\gamma^{\overline{1}} \cos \varphi+\gamma^{\overline{2}} \sin \varphi\right) \\
= & 0 \quad(\lambda=\varphi),
\end{aligned}
$$

so that

$\nabla_{\varphi} \gamma^{\lambda}=\nabla_{\varphi} \gamma^{t}+\nabla_{\varphi} \gamma^{r}+\nabla_{\varphi} \gamma^{\varphi}=0 \quad(\lambda=t, r, \varphi)$.

Therefore, Eqs. (A.10), (A.15), and (A.20), imply that

$\nabla_{\mu} \gamma^{\lambda}=0 \quad(\mu, \lambda=t, r, \varphi)$.

Thus, we have verified that the matrices $\gamma^{\mu}$, in the basis tetrad given in Eq. (6), are covariantly constant. 


\section{Appendix B: Derivation of the spin connection}

The spinor affine connection in Eq. (3) can be written more explicitly as

$$
\begin{aligned}
\Gamma_{t} & =\frac{1}{4} \omega_{t}^{\overline{0} \overline{1}}\left[\gamma_{0}, \gamma_{\overline{1}}\right]+\frac{1}{4} \omega_{t}^{\overline{0} \overline{2}}\left[\gamma_{\overline{0}}, \gamma_{\overline{2}}\right]+\frac{1}{4} \omega_{t}^{\overline{1} \overline{2}}\left[\gamma_{\overline{1}}, \gamma_{\overline{2}}\right], \\
\Gamma_{r} & =\frac{1}{4} \omega_{r}^{\overline{0} \overline{1}}\left[\gamma_{\overline{0}}, \gamma_{\overline{1}}\right]+\frac{1}{4} \omega_{r}^{\overline{0} \overline{2}}\left[\gamma_{\overline{0}}, \gamma_{\overline{2}}\right]+\frac{1}{4} \omega_{r}^{\overline{1} \overline{2}}\left[\gamma_{\overline{1}}, \gamma_{\overline{2}}\right], \\
\Gamma_{\varphi} & =\frac{1}{4} \omega_{\varphi}^{\overline{0} \overline{1}}\left[\gamma_{\overline{0}}, \gamma_{\overline{1}}\right]+\frac{1}{4} \omega_{\varphi}^{\overline{0} \overline{2}}\left[\gamma_{\overline{0}}, \gamma_{\overline{2}}\right]+\frac{1}{4} \omega_{\varphi}^{\overline{1} \overline{2}}\left[\gamma_{\overline{1}}, \gamma_{\overline{2}}\right], \\
\Gamma_{z} & =\frac{1}{4} \omega_{z}^{\overline{0} \overline{1}}\left[\gamma_{\overline{0}}, \gamma_{\overline{1}}\right]+\frac{1}{4} \omega_{z}^{\overline{0} \overline{2}}\left[\gamma_{\overline{0}}, \gamma_{\overline{2}}\right]+\frac{1}{4} \omega_{z}^{\overline{1} \overline{2}}\left[\gamma_{\overline{1}}, \gamma_{\overline{2}}\right] .
\end{aligned}
$$

In order to calculate the spin connection $\omega_{\mu \bar{a} \bar{b}}$ in Eq. (4), we use the Christoffel symbols given in Eqs. (A.4) and (A.5). Since $\omega_{t}^{\overline{0} \overline{1}}=\omega_{t}^{\overline{0} \overline{2}}=\omega_{t}^{\overline{1} \overline{2}}=0, \omega_{r}^{\overline{0} \overline{1}}=\omega_{r}^{\overline{0} \overline{2}}=\omega_{r}^{\overline{1} \overline{2}}=0$, and $\omega_{z}^{\overline{0} \overline{1}}=\omega_{z}^{\overline{0} \overline{2}}=\omega_{z}^{\overline{1} \overline{2}}=0$, we find $\Gamma_{t}=\Gamma_{r}=\Gamma_{z}=0$. Furthermore, we also can verify that $\omega_{\varphi}^{\overline{0} \overline{1}}=\omega_{\varphi}^{\overline{0} \overline{2}}=0$. As a result, we have

$\Gamma_{\varphi \nu}^{\mu} \neq 0$,

for $\mu=\varphi, v=r$ and $\mu=r, v=\varphi$. Thus, the only contribution for the spin connection is obtained from

$\omega_{\varphi}^{\overline{1} \overline{2}}=e_{\mu}^{\overline{1}} e^{\nu \overline{2}} \Gamma_{\varphi \nu}^{\mu}-e^{\nu \overline{2}} \partial_{\varphi} e_{\nu}^{\overline{1}}$,

which gives

$$
\begin{aligned}
\omega_{\varphi}^{\overline{1} \overline{2}}= & -\alpha r \sin \varphi \sin \varphi \frac{1}{r}+\cos \varphi \frac{1}{\alpha r} \cos \varphi\left(-\alpha^{2} r\right) \\
& -\sin \varphi \partial_{\varphi} \cos \varphi-\frac{1}{\alpha r} \cos \varphi \partial_{\varphi}(-\alpha r \sin \varphi) \\
= & 1-\alpha .
\end{aligned}
$$

Thus, for the specific basis tetrad (8), the connection is found to be

$\boldsymbol{\Gamma}=\left(0,0, \Gamma_{\varphi}, 0\right)$,

with the non-vanishing element given as

$\Gamma_{\varphi}=-\frac{1}{4}(1-\alpha)\left[\gamma_{\overline{1}}, \gamma_{\overline{2}}\right]=-\frac{1}{2}(1-\alpha) \gamma_{\overline{1}} \gamma_{\overline{2}}$.

\section{Appendix C: 2D Dirac oscillator}

In this appendix, we briefly discuss the usual 2D Dirac oscillator. We mention that although fully equivalent, the present construction is slightly different from the previous one in the literature [54]. Let us consider Eq. (13) with $\pi=\mathbf{p}-i M \omega \tilde{\beta} \mathbf{r}$. By using the representation for the $\tilde{\gamma}$ matrices in Eq. (16), we are left with

$\left[\sigma^{1} \pi_{1}+s \sigma^{2} \pi_{2}+\sigma^{3} M-E\right] \psi=0$,

with $\pi_{i}=p_{i}-i M \omega \sigma^{3} r_{i}$. By squaring Eq. (C.31), one obtains

$$
\left[p^{2}+M^{2} \omega^{2} r^{2}-2 M \omega\left(\sigma^{3}+s L_{3}\right)\right] \psi=\left(E^{2}-M^{2}\right) \psi .
$$

Equation (C.32), restoring the factors $\hbar$ and $c$, in terms of components, provides

$2 M c^{2}\left[H_{\mathrm{ho}}^{2 \mathrm{D}}-\hbar \omega-s \omega L_{3}\right] \psi_{1}=\left(E^{2}-M^{2} c^{4}\right) \psi_{1}$,

$2 M c^{2}\left[H_{\mathrm{ho}}^{2 \mathrm{D}}+\hbar \omega-s \omega L_{3}\right] \psi_{2}=\left(E^{2}-M^{2} c^{4}\right) \psi_{2}$,

where

$H_{\mathrm{ho}}^{2 \mathrm{D}}=\frac{p^{2}}{2 M}+\frac{1}{2} M \omega^{2} r^{2}$.

Equation (84) for $s=1$ agreed with the expressions found in Eq. (A2) of Ref. [55] and Eqs. (9) and (22) of Ref. [56]. Using the ansatz in Eq. (27) the energy eigenvalues are determined:

$E= \pm \sqrt{M^{2}+2 M \omega(2 n+|m|-s m)}$,

showing that the energy eigenvalues are spin dependent. It should be noted that for $s=1(s=-1)$ and $m>0(m<$ 0 ) the energy eigenvalues are independent of the quantum number $m$.

From Eq. (C.32), in the nonrelativistic limit $E=M+\mathscr{E}$ with $M \gg \mathscr{E}$, we have

$\left[H_{\mathrm{ho}}^{2 \mathrm{D}}-\omega\left(\sigma^{3}+s L_{3}\right)\right] \psi=\mathscr{E} \psi$.

The first term on the left side of Eq. (C.36) is the Hamiltonian of the nonrelativistic circular harmonic oscillator [57], explaining why this system is called the Dirac oscillator. The second term is a constant which shifts all energy levels. The last term is the spin-orbit coupling, which (restoring the factor $\hbar$ ) is of strength $\omega / \hbar$. Summarizing, the nonrelativistic limit of the 2D Dirac oscillator is the circular harmonic oscillator with a strong spin-orbit coupling term with all levels shifted by the factor $\omega$. Indeed, the shifted energy levels are

$\mathscr{E}+\omega=(1+2 n+|m|-s m) \omega$.

As for the relativistic case, for $s=1(s=-1)$ and $m>$ $0(m<0)$ the energy eigenvalues are independent of the quantum number $m$.

\section{References}

1. M. Moshinsky, A. Szczepaniak, J. Phys. A 22(17), L817 (1989). doi:10.1088/0305-4470/22/17/002

2. D. Dutta, O. Panella, P. Roy, Ann. Phys. 331(0), 120 (2013). doi:10. 1016/j.aop.2013.01.001

3. A. Bermudez, M.A. Martin-Delgado, A. Luis, Phys. Rev. A 77, 063815 (2008). doi:10.1103/PhysRevA.77.063815

4. A. Bermudez, M.A. Martin-Delgado, E. Solano, Phys. Rev. A 76, 041801 (2007). doi:10.1103/PhysRevA.76.041801

5. R.P. Martinez-y Romero, A.L. Salas-Brito, J. Math, Phys. 33(5), 1831 (1992). doi:10.1063/1.529660 
6. J. Bentez, R.P. Martnez y Romero, H.N. Núez-Yépez, A.L. SalasBrito, Phys. Rev. Lett. 64, 1643 (1990). doi:10.1103/PhysRevLett. 64.1643

7. O. Castaños, A. Frank, R. López, L.F. Urrutia, Phys. Rev. D 43, 544 (1991). doi:10.1103/PhysRevD.43.544

8. J. Grineviciute, D. Halderson, Phys. Rev. C 80, 044607 (2009). doi:10.1103/PhysRevC.80.044607

9. R. de Lima Rodrigues, Phys. Lett. A 372(15), 2587 (2008). doi:10. 1016/j.physleta.2007.12.070

10. J.P. Crawford, J. Math. Phys. 34(10), 4428 (1993). doi:10.1063/1. 530348

11. F. Vega, J. Math. Phys. 55(3), 032105 (2014). doi:10.1063/1. 4866914

12. S. Cai, T. Jing, G. Guo, R. Zhang, Int. J. Theor. Phys. 49(8), 1699 (2010). doi:10.1007/s10773-010-0349-7

13. J.A. Franco-Villafa ne, E. Sadurní, S. Barkhofen, U. Kuhl, F. Mortessagne, T.H. Seligman, Phys. Rev. Lett. 111(17), 170405 (2013). doi:10.1103/PhysRevLett.111.170405

14. P. Strange, Relativistic Quantum Mechanics: With Applications in Condensed Matter and Atomic Physics (Cambridge, Cambridge University Press, 1998)

15. F.M. Andrade, E.O. Silva, M.M. Ferreira Jr., E.C. Rodrigues, Phys. Lett. B 731, 327 (2014). doi:10.1016/j.physletb.2014.02.054

16. K. Bakke, C. Furtado, Ann. Phys. 336(0), 489 (2013). doi:10.1016/ j.aop.2013.06.007

17. J. Carvalho, C. Furtado, F. Moraes, Phys. Rev. A 84(3), 032109 (2011). doi:10.1103/PhysRevA.84.032109

18. A. Boumali, L. Chetouani, Phys. Lett. A 346(4), 261 (2005). doi:10. 1016/j.physleta.2005.08.002

19. M. Betrouche, M. Maamache, J.R. Choi, Adv. High Energy Phys. 2013(1â̆Ş10), 383957 (2013). doi:10.1155/2013/383957

20. C. Quesne, V.M. Tkachuk, J. Phys. A: Math. Gen. 38(8), 1747 (2005). doi:10.1088/0305-4470/38/8/011

21. K. Nouicer, J. Phys. A: Math. Gen. 39(18), 5125 (2006). doi:10. 1088/0305-4470/39/18/025

22. N. Ferkous, A. Bounames, Phys. Lett. A 325(1), 21 (2004). doi:10. 1016/j.physleta.2004.03.033

23. H. Belich, E.O. Silva, M.M. Ferreira Jr., M.T.D. Orlando, Phys. Rev. D 83(12), 125025 (2011). doi:10.1103/PhysRevD.83.125025

24. H.F. Mota, K. Bakke, Phys. Rev. D 89, 027702 (2014). doi:10. 1103/PhysRevD.89.027702

25. C. Filgueiras, F. Moraes, Phys. Lett. A 361(1-2), 13 (2007). doi:10. 1016/j.physleta.2006.09.030

26. M. Alford, J. March-Russell, F. Wilczek, Nucl. Phys. B 328(1), 140 (1989). doi:10.1016/0550-3213(89)90096-5

27. M.G. Alford, F. Wilczek, Phys. Rev. Lett. 62(10), 1071 (1989). doi:10.1103/PhysRevLett.62.1071

28. F.M. Andrade, E.O. Silva, M. Pereira, Phys. Rev. D 85(4), 041701(R) (2012). doi:10.1103/PhysRevD.85.041701

29. F.M. Andrade, E.O. Silva, M. Pereira, Ann. Phys. (N.Y.) 339(0), 510 (2013). doi:10.1016/j.aop.2013.10.001

30. E.R. Bezerra de Mello, J. High Energy Phys. 2004(06), 016 (2004). doi:10.1088/1126-6708/2004/06/016

31. S.M. Carrol, Spacetime and Geometry: An Introduction to General Relativity (Addison-Wesley, United States, 2003)
32. M. Pollock, Acta. Phys. Pol. B 41(8), 1827 (2010)

33. P. van Nieuwenhuizen, Phys. Rep. 68(4), 189 (1981). doi:10.1016/ 0370-1573(81)90157-5

34. I. Lawrie, A Unified Grand Tour of Theoretical Physics, 3rd edn. (Taylor \& Francis, London, 2012)

35. C.R. Hagen, Phys. Rev. Lett. 64(5), 503 (1990). doi:10.1103/ PhysRevLett.64.503

36. C.R. Hagen, Int. J. Mod. Phys. A 6, 3119 (1991). doi:10.1142/ S0217751X91001520

37. V.R. Khalilov, Eur. Phys. J. C 74(1), 2708 (2014). doi:10.1140/ epjc/s10052-013-2708-z

38. S. Albeverio, F. Gesztesy, R. Hoegh-Krohn, H. Holden, Solvable Models in Quantum Mechanics, 2nd edn. (AMS Chelsea Publishing, Providence, RI, 2004)

39. W. Bulla, F. Gesztesy, J. Math. Phys. 26(10), 2520 (1985). doi:10. $1063 / 1.526768$

40. F. Gesztesy, S. Albeverio, R. Hoegh-Krohn, H. Holden, J. Reine Angew. Math. 380(380), 87 (1987). doi:10.1515/crll.1987.380.87

41. L. Dabrowski, P. Stovicek, J. Math. Phys. 39(1), 47 (1998). doi:10. 1063/1.532307

42. R. Adami, A. Teta, Lett. Math. Phys. 43(1), 43 (1998). doi:10.1023/ A: 1007330512611

43. M. Reed, B. Simon, Methods of Modern Mathematical Physics. II. Fourier Analysis, Self-Adjointness. (Academic Press, New York, 1975)

44. M. Abramowitz, I.A. Stegun (eds.), Handbook of Mathematical Functions (Dover Publications, New York, 1972)

45. F.M. Andrade, E.O. Silva, T. Prudêncio, C. Filgueiras, J. Phys. G 40(7), 075007 (2013). doi:10.1088/0954-3899/40/7/075007

46. V.R. Khalilov, Theor. Math. Phys. 175(2), 637 (2013). doi:10.1007/ s11232-013-0052-y

47. V.R. Khalilov, Eur. Phys. J. C 73(8), 2548 (2013). doi:10.1140/ epjc/s10052-013-2548-x

48. V.R. Khalilov, C.L. Ho, Ann. Phys. (NY) 323(5), 1280 (2008). doi:10.1016/j.aop.2007.08.007

49. V.R. Khalilov, I. Mamsurov, Theor. Math. Phys. 161(2), 1503 (2009). doi:10.1007/s11232-009-0137-9

50. S.N.M. Ruijsenaars, Ann. Phys. (NY) 146(1), 1 (1983). doi:10. 1016/0003-4916(83)90051-9

51. C.R. de Oliveira, M. Pereira, J. Phys. A 43(35), 354011 (2010). doi:10.1088/1751-8113/43/35/354011

52. F.M. Andrade, E.O. Silva, Phys. Lett. B 719(4-5), 467 (2013).doi:10.1016/j.physletb.2013.01.062

53. D.K. Park, J. Math. Phys. 36(10), 5453 (1995). doi:10.1063/1. 531271

54. F.M. Andrade, E.O. Silva, Europhys. Lett. 108, 30003 (2014). doi:10.1209/0295-5075/108/30003

55. A. Bermudez, M.A. Martin-Delgado, A. Luis, Phys. Rev. A 77(3), 033832 (2008). doi:10.1103/PhysRevA.77.033832

56. N.A. Rao, B.A. Kagali, Mod. Phys. Lett. A 19(28), 2147 (2004). doi:10.1142/S0217732304014719

57. S. Flügge, Practical Quantum Mechanics (Classics in Mathematics) (Springer, Berlin, 1999) 